\title{
The Upper Bound on the Lightest CP Even Higgs Mass in the MSSM, NMSSM and ESSM
}

\author{
Partha Pratim Pal \\ Bolpur Sikshaniketan Ashram Vidyalaya, West Bengal, Bolpur, Birbhum, India
}

\section{Email address:}

partha4321@yahoo.com

\section{To cite this article:}

Partha Pratim Pal. The Upper Bound on the Lightest CP Even Higgs Mass in the Minimal Supersymmetric Standard Model (MSSM), Next to - Minimal Supersymmetric Standard Model (NMSSM) and Exceptional Supersymmetric Standard Model (ESSM). International Journal of High Energy Physics. Vol. 3, No. 1, 2016, pp. 1-4. doi: 10.11648/j.ijhep.20160301.11

\begin{abstract}
Recently a Higgs like particle is reported to be discovered at CMS and ATLAS experiments at CERN LHC with a mass of about $125 \mathrm{GeV}$. The aim of this paper is to study the theoretical upper bound of lightest CP even Higgs mass in MSSM, NMSSM and ESSM. Although at this moment it is not possible to conclude whether the observed Higgs like particle is Standard Model Higgs or Supersymmetric one, yet the present paper comes to the conclusion that it is possible to accommodate $\sim 125 \mathrm{GeV}$ Higgs mass within MSSM, NMSSM and ESSM.
\end{abstract}

Keywords: Higgs Mass, MSSM, NMSSM, ESSM

\section{Introduction}

The main reason for introducing low energy supersymmetric theories in particle physics was, in fact, their ability to solve the naturalness and hierarchy problems [1]. Supersymmetry prevents the Higgs boson mass from acquiring very large radiative corrections: the quadratic divergent loop contributions of the SM [2] particles to the Higgs mass squared are exactly cancelled by the corresponding loop contributions of their supersymmetric partners. This cancellation stabilizes the huge hierarchy between the GUT and electroweak scale and no extreme finetuning is required. In MSSM [3, 4, 5] every quark, lepton and gauge boson has a supersymmetric partner associated with it. MSSM requires two CP conserving Higgs doublets with opposite hypercharge so as to give masses to up-type and down-type fermions, by keeping consistency with supersymmetry and by avoiding gauge anomalies due to the fermionic super partners of the Higgs bosons. Recently a Higgs like particle is reported to be discovered at CMS and ATLAS experiments at CERN LHC with a mass of $\sim 125$ $\mathrm{GeV}$. In the Standard Model, the Higgs boson mass is usually considered as an adjustable parameter because the quartic coupling of the Higgs potential is arbitrary. In the MSSM, an intrinsic upper bound on the lightest Higgs boson mass is obtained from the quartic Higgs couplings which is no longer arbitrary but is constrained by SUSY. But MSSM contains a $\mu$-parameter in the superpotential where $\mu$ is a dimensionful parameter. It enters the Higgs potential with the soft scalar masses to determine the vacuum expectation value of the Higgs fields. This parameter has to be adjusted by hand to a value at the electroweak scale, in order to provide the correct pattern of electroweak symmetry breaking. This is a problem of the model and is called $\mu$-problem [6]. In the NMSSM [7, $8,9,10]$ an additional gauge singlet $\mathrm{S}$ is introduced which generates the $\mu$-term dynamically. In order to avoid a low energy global U(1) symmetry, the superpotential is also supplemented by a trilinear term $S^{3}$. However the super potential of the NMSSM remains invariant under a discrete $Z_{3}$ symmetry which, when broken at the weak scale, leads to the formation of domain walls in the early universe, which are inconsistent with modern cosmology. In an attempt to break the $Z_{3}$ symmetry, operators suppressed by powers of the Plank scale could be introduced. But these give rise to quadratically divergent tadpole contributions which would destabilize the mass hierarchy. The Exceptional Supersymmetric Standard Model (ESSM) $[11,12]$ is an $E_{6}$ inspired Supersymmetric model with an extra $U(1)_{N}$ gauge symmetry which solves the $\mu$ problem of the MSSM in a similar way to the NMSSM, but without the accompanying problems of singlet tadpoles or domain walls.

\section{Upper Bound on the Lightest CP even Higgs Mass in the MSSM}

The MSSM contains two Higgs doublets which give eight 
independent states. After three of them are used to give longitudinal components of the $\mathrm{W}$ and $\mathrm{Z}$ bosons, we have five physical states: two neutral scalars $h^{0}$ and $H^{0}$, a pseudoscalar $A^{0}$, and a pair of charged Higgs scalars $H^{ \pm}$. At the tree level their masses and couplings are determined by only two parameters - the ratio of the two vacuum expectation values $\tan \beta$ and the mass of the CP-odd Higgs boson usually taken to be $m_{A}$. The lightest CP-even Higgs mass is given by [13]:

$$
M_{h}^{2}=\frac{1}{2}\left(M_{A}^{2}+M_{Z}^{2}+\varepsilon^{\prime}\right)\left[1-\sqrt{1-\frac{4\left\{M_{Z}^{2} M_{A}^{2} \cos ^{2} 2 \beta+\varepsilon /\left(M_{A}^{2} \sin ^{2} \beta+M_{Z}^{2} \cos ^{2} \beta\right)\right\}}{\left(M_{A}^{2}+M_{Z}^{2}+\varepsilon^{\prime}\right)^{2}}}\right]
$$

The neutral scalar gets a large radiative correction from the top quark loop along with the top squark (stop) loop. To a approximation this contribution is given by

$$
\varepsilon=\frac{3 g^{2} m_{t}^{4}}{8 \pi^{2} M_{w}^{2}} \ln \left(\frac{M_{\tilde{t}}^{2}}{m_{t}^{2}}\right)
$$

Plus an additional contribution from the $\tilde{t}_{L, R}$ mixing, given by

$$
\varepsilon_{\text {mix }}=\frac{3 g^{2} m_{t}^{4}}{8 \pi^{2} M_{w}^{2}} \frac{A_{t}^{2}}{M_{\tilde{t}}^{2}}\left(1-\frac{A_{t}^{2}}{12 M_{\tilde{t}}^{2}}\right)
$$

where

$$
\varepsilon^{\prime}=\left(\varepsilon+\varepsilon_{m i x}\right) \frac{1+\tan ^{2} \beta}{\tan ^{2} \beta}
$$

The trilinear SUSY breaking parameter $A_{t}$ is given by

$$
A_{t}=\prod\left[\frac{\alpha_{i}\left(\mu_{t}\right)}{\alpha_{i}\left(m_{t}\right)}\right]^{\frac{c_{i}}{2 a_{i}}}
$$

where $t=\ln \beta, C_{i}=\left(\frac{17}{20}, \frac{9}{4}, 8\right) ; i=Y, 2 L, 3 C ; \mu_{t}>m_{t}$

$$
\begin{gathered}
\frac{1}{\alpha_{i}(\mu)}=\frac{1}{\alpha_{i}\left(m_{t}\right)}-\frac{a_{i}}{2 \pi} \ln \left(\frac{\mu_{t}}{m_{t}}\right) \\
i=1,2,3 ; a_{1}=\frac{21}{5}, a_{2}=-3, a_{3}=-7 \\
\mu_{t}=\sqrt{m_{t} M_{S U S Y}}=418.33 ; m_{t}=175 \mathrm{GeV}, M_{S U S Y} \\
=1000 \mathrm{GeV}, M_{\tilde{t}}=1000 \mathrm{GeV}, M_{w} \\
=80 \mathrm{GeV}, M_{Z}=91 \mathrm{GeV} \text { and } g=0.65
\end{gathered}
$$

Using equation (1) the variation of lightest Higgs boson mass $M_{h}$ is studied with systematic variation of $\tan \beta$ and $M_{A}$ for three different values of $m_{t}(=172,175$ and $180 \mathrm{GeV})$. The mass of $M_{h}$ increases with increase of $M_{A}$, until at about $300 \mathrm{GeV}$ for $M_{A}$, a maximum value of $M_{h}$ is reached for each value of $\tan \beta$. Beyond $M_{A}=300 \mathrm{GeV}, M_{h}$ is almost independent of $M_{A}$. For large values of the pseudoscalar Higgs boson mass, $M_{A} \gg M_{Z}$, the lightest Higgs boson mass $M_{h}$ reaches its maximum for a given value of $\tan \beta$. I have also studied the variation of $M_{h}$ with $m_{t}$ as $m_{t}$ varies from $172 \mathrm{GeV}$ to 180 $\mathrm{GeV}$. It is found that the value of $M_{h}$ increases with the increase of $m_{t}$. The value of $M_{h}$ for $m_{t}=180 \mathrm{GeV}$ gives the maximum value of $M_{h}$ which is $\sim 135 \mathrm{GeV}$. This is interesting because MSSM gives the upper bound on the lightest Higgs boson mass as $M_{h} \leq 135 \mathrm{GeV}$.

\section{Upper Bound on the Lightest CP- even Higgs Mass in the NMSSM}

The Higgs fields of the NMSSM consists of the usual two Higgs doublets as in the MSSM together with an extra Higgs singlet,

$$
H_{u}=\left(\begin{array}{c}
H_{u}^{+} \\
H_{u}^{0}
\end{array}\right), H_{d}=\left(\begin{array}{l}
H_{d}^{0} \\
H_{d}^{-}
\end{array}\right), S
$$

The extra singlet is allowed to couple only to the Higgs doublets of the model, and consequently the couplings of the new fields to gauge bosons will only be manifested via their mixing with the other Higgs fields.

The tree-level Higgs potential is composed of three parts [10],

$$
V=V_{F}+V_{D}+V_{S o f t}
$$

with

$$
\begin{gathered}
V_{F}=|\lambda S|^{2}\left(\left|H_{u}\right|^{2}+\left|H_{d}\right|^{2}\right)+\left|\lambda H_{u} H_{d}+K S^{2}\right|^{2} \\
V_{D}=\frac{g^{2}+g^{\prime 2}}{8}\left(\left|H_{d}\right|^{2}-\left|H_{u}\right|^{2}+\frac{1}{2} g^{2}\left|H_{u}^{+} H_{d}\right|^{2}\right) \\
V_{\text {Soft }}=m_{H_{u}}^{2}\left|H_{u}\right|^{2}+m_{H_{d}}^{2}\left|H_{d}\right|^{2}+m_{S}^{2}|S|^{2}+ \\
{\left[\lambda A_{\lambda} S H_{u} H_{d}+\frac{1}{3} K A_{K} S^{3}+\text { h.c. }\right]}
\end{gathered}
$$

Where $g$ and $g$ / being the gauge couplings of $S U(2)_{L}$ and $\mathrm{U}(1)$ interactions respectively. The Higgs potential contains seven parameters: $\lambda$ and $\mathrm{K}$ from the superpotential and $A_{K}, A_{\lambda}, m_{H_{u}}, m_{H_{d}}$ and $m_{S}$ from the soft supersymmetry breaking terms.

In the NMSSM seven Higgs bosons are created: $3 \mathrm{CP}$-even $\left(H_{1}^{0}, H_{2}^{0}, H_{3}^{0}\right)$, two CP-odd $\left(A_{1}^{0}, A_{2}^{0}\right)$ and two charged $\left(H^{ \pm}\right)$.

The tree level lightest CP-even Higgs mass in NMSSM is given by [10]. 


$$
M_{H_{1}}^{2}=\frac{1}{2}\left\{M_{Z}^{2}+\frac{1}{2} K v_{S}\left(4 K v_{S}+\sqrt{2} A_{K}\right)-\sqrt{\left[M_{Z}^{2}-\frac{1}{2} K v_{S}\left(4 K v_{S}+\sqrt{2} A_{K}\right)\right]^{2}+\cot ^{2} \beta_{S}\left[2 \lambda^{2} v_{S}^{2}-M_{A}^{2} \sin ^{2} 2 \beta\right]^{2}}\right\}
$$

where $v=\sqrt{v_{u}^{2}+v_{d}^{2}}=246 \mathrm{GeV}, \tan \beta=\frac{v_{u}}{v_{d}}, \tan \beta_{S}=\frac{v_{S}}{v}$ and negative value for $A_{K}$ being preferred.

The upper bound of the lightest CP-even Higgs mass comes out to be $\sim 140 \mathrm{GeV}$. It is reached for

$$
\begin{gathered}
\tan \beta \sim 2.2, \lambda \sim 0.677, k \sim 0.068, v_{s}=15 v, M_{A}=1000 \mathrm{GeV}, m_{Z} \\
=175 \mathrm{GeV}, \mu \sim 545 \mathrm{GeV}, A_{\lambda} \sim 1365 \mathrm{GeV} \text { and } A_{K} \sim 10 \mathrm{GeV} .
\end{gathered}
$$

\section{Upper Bound on the Lightest CP- even Higgs Mass in the ESSM}

The Exceptional Supersymmetric Standard Model (ESSM) represents a low energy alternative to the MSSM or NMSSM and provides a solution to the $\mu$ problem without domain wall problem. ESSM involves the low energy matter content of three 27 representations of $E_{6}$, which is broken at the GUT scale, and allows gauge coupling unification due to an additional pair of Higgs-like doublets. The ESSM predicts a $Z^{\prime}$ boson and exotic quarks which, if light enough, will provide spectacular new physics signals at the LHC. Within the $E_{6}$ inspired supersymmetric standard model there is a unique choice of Abelian gauge group, referred to as $U(1)_{N}$, which allows zero charges for right-handed neutrinos. This choice of $U(1)_{N}$ allows large right-handed neutrino Majorana masses and hence a high scale see-saw mechanism. A rich spectrum of new states at the $\mathrm{TeV}$ scale is predicted by $E_{6} S S M$ corresponding the matter content of three 27 component families. Since each 27 includes a pair of Higgs doublets plus a SM singlet, the $E_{6} S S M$ predicts a total three families of Higgs doublets and three families of Higgs singlets. The two Higgs doublets familiar from the MSSM are denoted as $H_{d}$ and $H_{u}$, while the two further replicas of these Higgs doublets predicted by the $E_{6} S S M$ are denoted as $H_{1}^{d}, H_{1}^{u}$ and $H_{2}^{d}, H_{2}^{u}$. Each 27 representation also contains a separate SM singlet. There is the singlet $\mathrm{S}$ whose VEV yields an effective $\mu$ term, plus two further copies of this singlet, $S_{1}$ and $S_{2}$.

$E_{6} S S M$ Higgs fields develop the VEVs $\left\langle H_{d}\right\rangle=\frac{v_{d}}{\sqrt{2}},\left\langle H_{u}\right\rangle=$ $\frac{v_{u}}{\sqrt{2}}$ and $\langle s\rangle=\frac{s}{\sqrt{2}}$, thus breaking the $S U(2)_{w} \times U(1)_{Y} \times U(1)_{N}$ symmetry to $U(1)_{E M}$. After Electro-Weak symmetry breaking two $\mathrm{CP}$-odd and two charged Goldstone models in the Higgs sector are absorbed by the $Z, Z^{\prime}$ and $W^{ \pm}$gauge bosons so that only six physical degrees of freedom are left. They form one CP-odd, two charged and three CP-even Higgs states.

The tree-level upper bound on the lightest CP-even Higgs boson is given by,

$$
m_{h_{1}}^{2} \lesssim \frac{\lambda^{2}}{2} v^{2} \operatorname{Sin}^{2} 2 \beta+M_{Z}^{2} \operatorname{Cos}^{2} 2 \beta+\frac{M_{Z}^{2}}{4}\left(1+\frac{1}{4} \operatorname{Cos} 2 \beta\right)^{2}
$$

From equation (10) it is clear that, at tree level the theoretical upper bound on the lightest Higgs mass in the ESSM depends on $\lambda$ and $\tan \beta$ only. The requirement of validity of perturbation theory up to the Grand Unification Scale constrains the interval of variations of the Yukawa coupling $\lambda$ for each value of $\tan \beta$. The resulting tree-level upper bound on the mass of the lightest Higgs particle in the ESSM attains a maximum value of $130 \mathrm{GeV}$ at $\tan \beta=1.5-1.8$.

\section{Discussions and Conclusions}

The theoretical upper bound on the lightest CP even Higgs mass in the MSSM, NMSSM, and ESSM comes out to be $\sim 135 \mathrm{GeV}, \sim 140 \mathrm{GeV}$ and $130 \mathrm{GeV}$ respectively. Recently $\sim 125 \mathrm{GeV}$ Higgs like particle has been discovered at CMS and ATLAS experiments at CERN. In this context a very important question arises: Is the scalar Higgs boson observed at the LHC, a supersymmetric Higgs boson or a Standard Model one? The present paper throws light on the fact that it is possible to accommodate $\sim 125 \mathrm{GeV}$ Higgs mass within MSSM, NMSSM, and ESSM. The problem of resolving the issue of newly discovered boson may be understood through independent discovery, with caution in accepting new ideas and after critical enquiry into the evidence of discovery of the Higgs boson.

\section{References}

[1] E. Witten, Nucl. Phys. B188 (1981) 513; ibid Nucl. Phys. B202 (1982) 253; N. Sakai, Z. Phys. C11 (1981) 153; S. Dimopoulos and H. Georgi, Nucl. Phys. B193 (1981) 150; R. K. Kaul and P. Majumdar, Nucl. Phys. B199 (1982) 36.

[2] Glashow S L, Nucl. Phys. 22, 1961, 579; Salam A and Ward J C, Phys. Lett. 13, 1964, 168; Weinberg S, Phys. Rev. Lett. 19, $1967,1264$.

[3] Veltman M, Acta Phys. Polon. B12 (1981) 437; Witten E, Nucl. Phys. B 188 (1981) 513 and Phys. Lett. B 105 (1981) 267.

[4] Gildener E and Weinberg S, Phys. Rev. D 13, 3333 (1976); Susskind L, Phys. Rev. D 20 (1979) 2619; Weinberg S, Phys. Lett. B 82 (1979) 387; t Hoof G, in Recent developments inGauge Theories, Proceedings of the NATO Advanced Study Institute, Cargese, 1979, eds., tHooft $\mathrm{G}$ et. al. (Plenum Press, NY, 1980).

[5] Gunion J F, Haber H E, Kane G and Dawson S, The Higgs Hunters' Guide (Addition-Weslay, Reading, MA, 1990); Haber H E and Kane G, Phys. Rep. 117, 75 (1985). and references therein for SM; Introduction to supersymmetry (http://arxiv.org/pdf/hep-th/9612114) by Lykken J D, 1996; An introduction to supersymmetry (http://arxiv.org/pdf/hepph/9611409)by Drees M, 1996; A Supersymmetry Primer (http://arxiv.org/pdf/hep-ph/9709356) By Martin S, 1999; Introduction to Supersymmetry (http://arxiv.org/pdf/hepph/0101055) By Bilal A, 2001; An introduction to Global supersymmetry.

(http://www.physics.uc.edu/ argyres/661/susy2001.pdf) By Philip Arygres, 2001.

[6] J. E. Kim and H. P. Nilles, Phys. Lett. B 138, 150 (1984). 
4 Partha Pratim Pal: The Upper Bound on the Lightest CP Even Higgs Mass in the Minimal Supersymmetric Standard Model (MSSM), Next - to - Minimal Supersymmetric Standard Model (NMSSM) and Exceptional Supersymmetric Standard Model (ESSM)

[7] M. Bastero-Gil, C. Hugonic, S. F. King, D. P. Roy and S. Vempati, Phys. Lett. B 489, 359 (2000); A. de Gouvea, A. Friedland and H. Murayama, Phys. Rev. D 57, 5676 (1998).

[8] J. Ellis, J. F. Gunion, H. Haber, L. Roszkowski, F. Zwirner, Phys. Rev. D 39 (1989) 844.

[9] J. P. Derendinger and C. A. Savoy, Nucl. Phys. B 237 (1984) 307; J. M. Frere, D. R. Jones and S. Raby, Nucl. Phys. B 222 (1983) 11
[10] P. P. Pal, Intertational Journal of High Energy Physics, 2015; 2 (3): 34-37, DOI: 10.11648/j.ijhep.20150203.11.

[11] S. F. King, S. Moretti, R. Nevzorov, arXiv: hep-ph/0510419.

[12] S. F. King, S. Moretti, R. Nevzorov, arXiv: hep$\mathrm{ph} / 0601269 \mathrm{v} 2$.

[13] S. Chakrabarty and P. P. Pal, J. Assam Sc. Soc, Vol. 51, June 2010, pp. 18-26. 